\title{
Information systems capabilities and organizational agility: Understanding the mediating role of absorptive capacity when influenced by a hierarchy culture
}

\author{
Completed Research Paper
}

\author{
José L. Roldán \\ Universidad de Sevilla \\ jlroldan@us.es \\ Carmen Felipe \\ Universidad de Sevilla \\ cfelipe@us.es
}

\author{
Antonio L. Leal-Rodríguez \\ Universidad Loyola Andalucía \\ alleal@uloyola.es
}

\begin{abstract}
Organizational agility (OA), as a key dynamic capability, is a firm's ability to enable sensing environmental changes and responding efficiently and effectively to them. This study explores this topic further by analyzing the part played by the information systems capabilities (ISC) variable as an antecedent of OA, and absorptive capacity (AC) as a mediator construct. Furthermore, we test the negative moderating role of hierarchy culture (HC) in the AC-OA link. Using partial least squares (PLS) and the PROCESS macro, we find evidence of these relations proposed, and the existence of a conditional mediating situation generated by $\mathrm{HC}$.
\end{abstract}

Keywords: organizational agility, information systems capabilities, absorptive capacity, hierarchy culture, partial least squares (PLS), mediating analysis, moderating effect, conditional mediation model.

\section{Introduction}

Organizations are currently facing highly turbulent environments, which are mainly characterized by strong doses of dynamism, complexity and uncertainty. These conditions have led to hypercompetitive markets where the survival of companies is certainly threatened. In such a context, knowing the mechanisms that allow organizations to detect, adapt and offer the proper response to the environment's changes becomes especially relevant, as this might lead firms to attain a greater success by exploiting emerging opportunities and new sources of competitive advantages. Therefore, the concept of organizational agility (OA) appears as a key issue concerning organizational survival and success.

Agile organizations are those that are able to effectively operate within hypercompetitive, unpredictable and constantly changing environments (Goldman, Nagel \& Preiss, 1995). Thus, OA can be defined as the firm's capability to sense the changes of the environment and respond efficiently and effectively to them (Ashrafi et al., 2005). Assuming the dynamic capabilities theory as a reference framework (Teece, Pisano, \& Shuen, 1997), OA is identified as one of the key dynamic capabilities for organizations in order to achieve sustainable competitive advantages (Sambamurthy, Bharadwaj, \& Grover, 2003) and to survive in highly dynamic environments (Nijssen \& Paauwe, 2012). 
This topic has been attracting the attention of academic research since the mid-1990s and has been assessed by multiple disciplines. This has led to successive limitations of the concept of agility, removing it from its general aspect of organizational capacity (Charbonnier-Voirin, 2011). In addition, over the last years, the focus of research on the technological aspect of business has led to forgetting other contextual organizational factors equally or even more relevant, such as culture, communication and leadership (Crocitto \& Youssef, 2003). Basically, the core of these studies has been the role of information systems capabilities (ISC) in achieving a higher level of OA.

This paper hence aims to cover such research gaps inherent to OA that the prior literature has until now failed to do. Therefore, we mean to answer the following questions: (1) From an inclusive point of view, what is OA? (2) What are the antecedents of OA? Can we consider other variables apart from ISC (e.g., absorptive capacity)? (3) What are the links between such antecedents? Do ISC affect OA directly or through an indirect relationship? (4) Could the presence of certain cultural values become a moderator of the aforementioned relation?

The paper proceeds as follows. The next section presents the theoretical background together with the research model and hypotheses. The third section comprises a description of the research methodology. The fourth section presents the results of the different data analyses carried out. Finally, we bring together the discussion and implications.

\section{Literature Review and Research Hypotheses}

\subsection{Approaching the concept of organizational agility}

The concept of organizational agility has its roots in two prior related concepts: organizational adaptability (a reactive aspect) and organizational flexibility (a proactive aspect) (Sherehiy, Karwowski, \& Layer, 2007). In this vein, OA involves a firm's ability to sense and respond to environmental changes (Overby, Bharadwaj, \& Sambamurthy, 2006). The contribution of Sambamurthy et al. (2003) is quite remarkable. They state that OA comprises three interrelated dimensions: customer agility (leveraging the voice of customers to gain market intelligence), partnering agility (learning from business partners to enhance the firm's response to the market) and operational agility (rapid process redesign to exploit dynamic marketplace conditions). Therefore, following Charbonnier-Voirin (2011), we define $\mathrm{OA}$ as the intentional response capability developed by the organization to enable it to act efficiently in a highly turbulent environment, not only by reacting rapidly to change, but also through its potential of action in anticipating and seizing opportunities, in particular through innovation and learning.

\subsection{The relationship between information systems capabilities and organizational agility}

The concept of information systems capabilities (ISC) comes from the use of the resourcebased theory in the information technologies (IT) research field. This theory enabled the establishment of a framework to assess the strategic contribution of information systems (IS) resources to the company (Wade \& Hulland, 2004). Under such a perspective, the firm's IS resources (assets and capabilities) that are inimitable and valuable may lead to achieving sustained competitive advantages (Ravichandran \& Lertwongsatien, 2005).

Bharadwaj (2000) defines ISC as the firm's abilities to mobilize and deploy IT-based resources in combination or jointly with other resources and capabilities. These are skills, competences and abilities upon which the value of the physical IT resources can be leveraged (Doherty \& Terry, 2009). Wade and Hulland (2004) describe three types of ISC: inside-out 
(deployed from inside the firm in response to market requirements and opportunities), outside-in (externally oriented, placing an emphasis on anticipating market requirements), and spanning capabilities (needed to integrate the firm's inside-out and outside-in capabilities).

There is a lack of consensus in the scientific literature concerning whether the impact of ISC on OA is positive or negative. On the one hand, some researchers supporting the negative impact argue that limitations of inflexible IT systems may result in a rigidity which hinders or even impedes the adaptation to the environment's requirements (Overby et al., 2006). On the other hand, Sambamurthy et al. (2003) consider that ITs are generators of the digital options through which OA is positively affected. This is not only because they allow the creation of new information-based products and services, but also because they enable the coordination of internal processes and the building of new interorganizational relationships. Lu and Ramamurthy (2011) show that ISC have a direct effect on agility, indicating that firms need to continually develop superior IT capabilities in order to successfully manage and exploit their resources, with the aim of building agile organizations.

With the support of this line of the literature, we posit that properly deployed and managed ISC can provide tools and instruments for organizations to enhance their capabilities to sense and respond to environmental changes. Therefore we postulate the following hypothesis:

H1: The firm's information systems capabilities (ISC) are positively linked to its organizational agility $(O A)$.

\subsection{The mediating role of absorptive capacity in the relationship between ISC and OA.}

Within the current dynamic environment, organizational learning has become a key success factor for firms. The study of absorptive capacity (AC) is hence perfectly embedded within such a scenario. Cohen and Levinthal (1990) initially defined AC as the firm's ability to recognize the value of new external knowledge, assimilate it and apply it to commercial ends. Zahra and George (2002) later developed an extension of the AC concept, broadly defining it as a set of organizational routines and processes through which firms acquire, assimilate, transform and exploit knowledge in order to produce a dynamic organizational capability. These four activities are complementary and build upon each other to produce AC.

Liu, Ke, Wei, and Hua (2013) indicate that ISC are key factors for the development of higher order capabilities, such as the AC. In fact, some IS functions, such as developing knowledge repositories, effective information retrieving mechanisms, or enabling collaboration and communication between knowledge producers (experts) and knowledge seekers, play a key role in the firm's AC enhancement (Ashrafi, Xu, Kuilboer, \& Koehler, 2006). In this vein, Cepeda-Carrión, Cegarra-Navarro and Jiménez-Jiménez (2012) consider that ISC support organizations' AC, since they enable new knowledge to be combined with past knowledge in order to be exploited.

There is still is a gap for researchers concerning the tie between AC and OA. Regarding OA, Lu and Ramamurthy (2011) suggest as a future research line the study of the mechanisms for developing routines and structures that facilitate learning and experimentation, and improve capacity building. A company with a stronger AC is more prepared to perceive changes in the markets and to learn from experience (Malhotra, Gosain, \& Sawy, 2005). Ashrafi et al. (2005) state that there are no empirical studies in the literature to explain how and why investment in knowledge acquisition drives OA, and propose two concepts - AC and dynamic capabilities - as enablers for achieving agility. Hao, Yu and Dong (2011) point out the mediating role of AC by its translating knowledge management systems usage into higher order organizational capabilities, i.e., agility and innovativeness

To sum up, ISC lead to an enhanced AC, and a greater AC might improve the agility of 
the organization. Based on this logic and previous research, we thus hypothesize:

H2: The relation between information systems capabilities (ISC) and organizational agility $(O A)$ is mediated by absorptive capacity $(A C)$.

\subsection{The moderating effect of a hierarchy culture in the relationship between $A C$ and $O A$.}

Different organizational values generate disparate knowledge management (KM) behaviors and these will lead to varying outcomes (Alavi, Kayworth \& Leidner, 2006). While some cultural values, such as openness and trust, can induce positive KM behaviors (e.g., knowledge contribution and sharing), which will lead to innovation, there are other values that might lead to dysfunctional KM behaviors (e.g., information hoarding) and, hence, negative results such as organizational rigidity. This means organizational culture may become a barrier that hinders $\mathrm{AC}$ and its effects if it remains excessively rigid and controloriented.

Using the competing values framework (Cameron \& Quinn, 2011), we have focused on the hierarchy culture (HC), which is often labeled as bureaucratic, top-down focused, ruleoriented, and by-the-book (Zammuto, Gifford \& Goodman, 2000). This cultural typology is based upon minimal ambiguity levels and an excessive sense of safety, predictability, efficiency, stability, uniformity, etc. In short, it can be sustained that HC is a cultural typology essentially oriented toward efficiency and internal control. Moreover, its values are internally focused and are thus more aligned with keeping a static and rigid hierarchical structure than pursuing business opportunities in the market. We hence hypothesize:

H3: Hierarchy culture (HC) moderates (decreasing) the link between absorptive capacity $(A C)$ and organizational agility $(O A)$.

\section{Research Method}

\subsection{Sample and data collection}

We have chosen innovative classified sectors as the population for this study. These industries can be considered as hypercompetitive, requiring a flexible and quick response from organizations. The sector selection has been carried out using the classification developed by the Spanish National Institute of Statistics (Cotec, 2009) of high and mediumhigh technology industries. This generated a population of 2,360 firms. An off-line survey was used to gather data. Because the level of analysis is the organization, the respondent of the questionnaire was a senior management member. After one mailing effort, the outcome was 172 usable surveys (a $7.3 \%$ response rate). The organizations participating belonged primarily to the computer systems design (26.7\%), machinery manufacturing (18\%) and chemical $(17.4 \%)$ sectors. Other industries were included in the manufacturing sectors of transportation equipment $(8.1 \%)$, electrical equipment $(7.6 \%)$, and computer and electronic products (7\%). According to the European Union classification, $23.8 \%$ of the firms participating were large enterprises, with more than 250 employees. Of the respondents, $23.8 \%$ belonged to the research and development department, followed by the marketing department $(20.9 \%)$, general management (14\%), and the engineering department $(9.3 \%)$. Most of the respondents were male (66\%), whereas women represented $34 \%$.

\subsection{Measures}

The review of the literature allowed us to identify validated measures for each construct. 
Our efforts focused on making the necessary adjustments to the context of the study (i.e., the Spanish language and setting). A pilot test of the survey was conducted in order to assess the content validity. The ISC variable, as a superordinate multidimensional construct, was measured by eight items adapted from Wade and Hulland (2004). To assess AC as a superordinate multidimensional construct, we adapted 21 items from Jansen, Van Den Bosch and Volberda (2005). OA was also modeled as a superordinate multidimensional construct and measured by eleven items adapted from Lu and Ramamurthy (2011), Yang and Liu (2012), Bradley, Pratt, Byrd, Outlay, and Wynn (2012), and Tallon and Pinsonneault (2011). The measurement of the $\mathrm{HC}$ variable used an adaptation of the scale appearing in Cameron and Quinn (2011). This variable has been modeled as a unidimensional construct shaped by six reflective items. Finally, we controlled the size (number of employees) and the age (number of years since the founding) of the firm. All the variables were measured on the basis of seven-point Likert scales, except controls.

\subsection{Data analysis}

We have used Partial Least Squares (PLS) path modeling to test the research model. The choice of PLS is based on the following reasons (Roldán \& Sánchez-Franco, 2012): (1) The focus of the study is the prediction of the dependent variables; (2) the sample $(n=172)$ is not very large; (3) the research model is complex according to the type of relationships (direct, mediated and moderated) described in the hypotheses and the levels of dimensionality; (4) this study uses latent variables scores in the subsequent analysis of predictive relevance, particularly in the implementation of the two-stage approach for modeling multidimensional constructs (Wright, Campbell, Thatcher, \& Roberts, 2012); and (5) the nature of most theoretical constructs is defined, as we rely on a composite measurement model with a reflective design approximation, which means that indicators and dimensions represent different facets but are expected to be correlated (Henseler, 2014). This way, constructs are modeled as composites of their indicators without error term (Fornell, 1982). This study uses SmartPLS v. 3.2 software (Ringle, Wende, \& Becker, 2015) for the PLS analysis, and PROCESS macro 2.13 (Hayes, 2013) for the moderated mediation analysis.

\section{Results}

\subsection{Measurement model}

First, the indicators and dimensions satisfy the requirement of reliability since their loadings are, in general, greater than 0.7 (Table 1). In order to accomplish this result, we carried out an item trimming process with some weak items of the AC instrument. In addition, some items of the HC construct also had weak loadings. Notwithstanding, we have decided to retain them in order to support the content validity of the scale. Due to size limitations, we only show loadings for dimensions.

Second, all multidimensional constructs and dimensions meet the requisite of construct reliability, because their composite reliabilities (CR) are greater than 0.7. Third, these latent variables attain convergent validity since their average variance extracted (AVE) surpasses the 0.5 level or are very near to it (Table 1). Lastly, Table 2 shows that all variables achieve discriminant validity following both the Fornell-Larcker and the HTMT ${ }^{90}$ criterion, however, the $\mathrm{AC}$ and $\mathrm{OA}$ variables may have a discriminant validity problem according to the HTMT ${ }^{.85}$ criterion (Henseler, Ringle \& Sarstedt, 2014). 
Table 1: Measurement model results

\begin{tabular}{lccc}
\hline Construct/Dimension & Loading & CR & AVE \\
\hline Information systems capabilities (SMC) & & $\mathbf{0 . 9 3 4}$ & $\mathbf{0 . 8 2 4}$ \\
Outside-in capabilities & 0.876 & 0.908 & 0.832 \\
Spanning capabilities & 0.937 & 0.935 & 0.878 \\
Inside-out capabilities & 0.910 & 0.940 & 0.796 \\
Absorptive capacity (SMC) & & $\mathbf{0 . 8 9 9}$ & $\mathbf{0 . 6 9 1}$ \\
Acquisition & 0.763 & 0.839 & 0.511 \\
Assimilation & 0.802 & 0.892 & 0.735 \\
Transformation & 0.919 & 0.862 & 0.611 \\
Exploitation & 0.834 & 0.793 & 0.563 \\
Organizational agility (SMC) & & $\mathbf{0 . 9 2 1}$ & $\mathbf{0 . 7 9 5}$ \\
Operational agility & 0.860 & 0.911 & 0.773 \\
Customer agility & 0.946 & 0.912 & 0.776 \\
Partnering agility & 0.866 & 0.885 & 0.611 \\
Hierarchy culture (reflective construct) & & $\mathbf{0 . 8 5 1}$ & $\mathbf{0 . 4 9 2}$ \\
\hline Niter: cons
\end{tabular}

Notes: CR: composite reliability; AVE: Average variance extracted; SMC: superordinate multidimensional construct

Table 2: Measurement model. Discriminant validity

\begin{tabular}{ccccccccccccccc}
\hline & \multicolumn{4}{c}{ Fornell-Larcker Criterion } & \multicolumn{4}{c}{ Heterotrait-Monotrait Ratio (HTMT) } \\
\hline & ISC & AC & OA & HC & Age & Size & & ISC & AC & OA & HC & Age & Size \\
\hline ISC & $\mathbf{0 . 9 0 8}$ & & & & & & ISC & & & & & & & \\
AC & 0.665 & $\mathbf{0 . 8 3 1}$ & & & & & AC & 0.761 & & & & & \\
OA & 0.638 & 0.765 & $\mathbf{0 . 8 9 2}$ & & & & OA & 0.716 & 0.885 & & & & \\
HC & 0.455 & 0.467 & 0.503 & $\mathbf{0 . 7 0 1}$ & & & HC & 0.508 & 0.504 & 0.548 & & & \\
Age & 0.064 & 0.099 & 0.144 & 0.157 & n.a. & & Age & 0.069 & 0.102 & 0.151 & 0.175 & & \\
Size & 0.199 & 0.131 & 0.132 & 0.125 & 0.327 & n.a. & Size & 0.212 & 0.141 & 0.137 & 0.131 & 0.327 & \\
\hline
\end{tabular}

Notes: ISC: information systems capabilities; AC: absorptive capacity; OA: organizational agility; HC: hierarchy culture. Fornell-Larcker Criterion: Diagonal elements (bold) are the square root of the variance shared between the constructs and their measures (AVE). Off-diagonal elements are the correlations among constructs. For discriminant validity, diagonal elements should be larger than off-diagonal elements. n.a.: non-applicable.

\subsection{Structural model}

Table 3 includes the main parameters obtained for the four models under study in the structural assessment. Model 1 describes the significant total effect $\left(c=0.642^{* * *}\right)$ of ISC on OA once the effect of controls (age and size) has been considered. Model 2 shows how the direct effect of ISC on OA decreases, although it remains significant $\left(c^{\prime}=0.236^{* * *}\right)$, when AC is included. This supports H1. Furthermore, paths $a$ and $b_{1}$ are significant. Therefore, both the decrement manifested in the direct effect $\left(c^{\prime}\right)$ and the significance of the regression coefficients $a$ and $b_{1}$ would be suggesting the potential existence of an indirect effect of ISC on $\mathrm{OA}$ via $\mathrm{AC}$ as a mediator (H2). Nonetheless, the key condition to determine such a mediating effect is to test the significance of $a \times b_{1}$ (Hayes, 2009). With this aim in mind, we have obtained the value for this indirect effect $\left(a \times b_{1}=0.401\right)$ from SmartPLS, which is significant (Table 4). This output supports H2. Consequently, we assume a partial mediation of the AC in the relation between ISC and OA since the direct $\left(\mathrm{H} 1=c^{\prime}\right)$ and the indirect $(\mathrm{H} 2$ $=a \times b_{1}$ ) effects are both significant (Baron \& Kenny, 1986). In addition, we have calculated the variance accounted for (VAF) index (Hair, Hult, Ringle, \& Sarstedt, 2014), which determines the size of the indirect effect $\left(a \times b_{1}\right)$ in relation to the total effect $(c)$. When the VAF has an outcome between $20 \%$ and $80 \%$, a partial mediation can be expected. This occurs in our case, given that the VAF for the indirect effect is $62.45 \%$ (Table 4). Finally, we have sought to take a further step forward by computing the standardized root mean square residual (SRMR), as the root mean square discrepancy between the correlations observed and the model-implied correlations (Hu \& Bentler, 1999) for the model with the total effect and the 


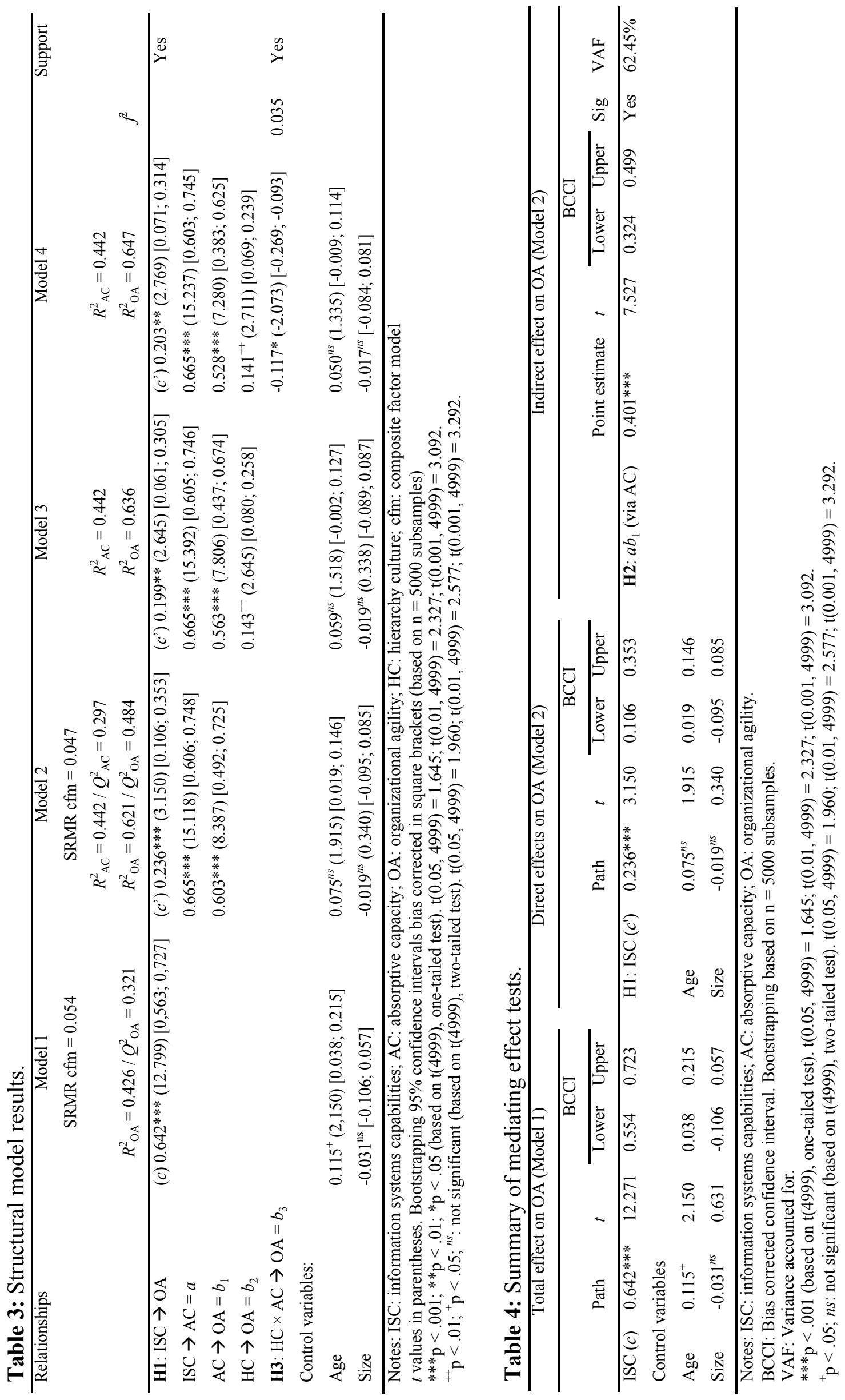


model with the indirect effect. Following Henseler et al. (2014), we have determined the SRMR for a composite factor model. This provides the exact fit of the composite factor model, thus constituting a confirmatory composite analysis. Model 1 (total effect) achieves a SRMR composite factor model of 0.054 , which means an appropriate fit assuming the usual cut-off of 0.08 (Hu \& Bentler, 1999). However, the SRMR composite factor model for Model 2 is still better: 0.047 . This would imply an additional support for the mediating role of AC.

The moderation hypothesis (H3: $\left.b_{3}\right)$ of the hierarchy culture (HC) in the path between AC and OA is tested using the product-indicator technique (Chin, Marcolin, \& Newsted, 2003). Model 3 includes $\mathrm{HC}$ and Model 4 adds the interaction term $\left(\mathrm{HC} \times \mathrm{AC}=b_{3}\right)$ (Table 3). The result seems to support H3 $\left(b_{3}=-0.117^{*}\right)$ (Table 3, Model 4) (Figure 1). Moreover, the overall effect size for $b_{3}$ achieves an $f^{2}$ value of 0.035 , which exceeds the minimum threshold of 0.02 (Chin, Marcolin, \& Newsted, 2003).

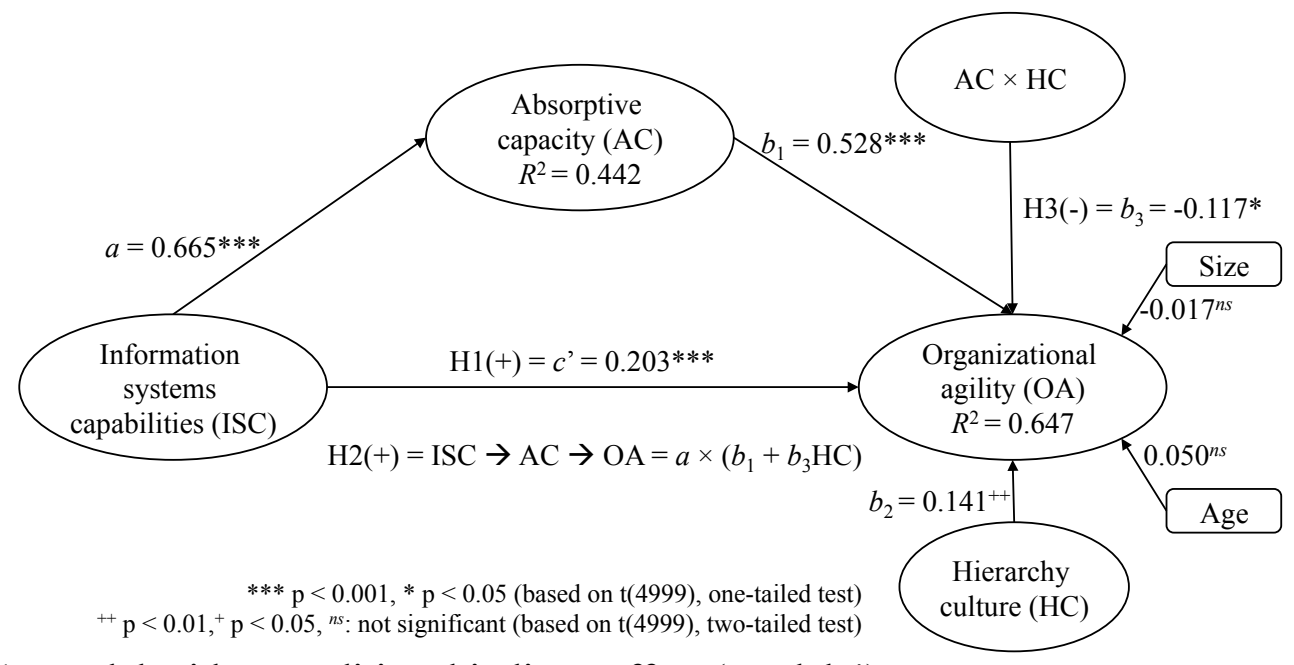

Figure 1: Model with a conditional indirect effect (Model 4)

The support for $\mathrm{H} 3$ together with the significant indirect effect $\left(a \times b_{1}\right)$ generates the emergence of a moderated mediation (Hayes, 2013). This involves the dependence of the indirect effect $\left(a \times b_{1}\right)$ on the value of $\mathrm{HC}\left(b_{3}\right)$, which would act as a moderator variable. As AC's effect on OA is contingent on the HC variable, so is ISC's indirect influence on OA. Following Hayes (2013), such an indirect impact is $a \times\left(b_{1}+b_{3} \mathrm{HC}\right)$.

In order to estimate this conditional indirect effect, we have applied the PROCESS macro developed by Hayes (2013). Using latent variable scores from SmartPLS 3 as input, PROCESS produces estimates and bias-corrected 95\% bootstrap CI for the indirect effect at different values of $\mathrm{HC}$ as a moderating construct. Table $5 \mathrm{~A}$ shows that the indirect effect of ISC on OA through AC is consistently positive and decreases as the HC values increase. A 95\% CI bias-corrected bootstrap for the conditional indirect effect is above zero for the different values of HC. This indirect impact is significant in all the scenarios analyzed. Hence, AC partially mediates ISC's influence on OA, although this indirect effect decreases as HC increases its value. Finally, Table 5B contains an index of moderated mediation $(-0.0512)$ (Hayes, 2015), which is also significant.

Last of all, the significant direct effect (non-hypothesized) of HC on OA (Models 3 and 4, Table 3) deserves to be commented on. In spite of its negative moderating influence on the path between AC and OA (Model 4), we find some evidence of a positive influence of the values associated with $\mathrm{HC}$ on the $\mathrm{OA}$ achieved by firms. 
Table 5: Conditional indirect effect analyses

\begin{tabular}{|c|c|c|c|c|c|}
\hline \multicolumn{6}{|c|}{ A) Conditional indirect effect of ISC on OA at values of HC as moderator } \\
\hline \multirow[b]{2}{*}{ Mediator } & \multirow[b]{2}{*}{$\mathrm{HC}$} & \multirow[b]{2}{*}{ Effect } & \multirow[b]{2}{*}{ Boot SE } & \multicolumn{2}{|c|}{ BCCI } \\
\hline & & & & Lower & Upper \\
\hline $\mathrm{AC}$ & -1.0029 & 0.4079 & 0.0604 & 0.3025 & 0.5412 \\
\hline $\mathrm{AC}$ & 0 & 0.3565 & 0.0596 & 0.2531 & 0.4877 \\
\hline $\mathrm{AC}$ & 1.0029 & 0.3051 & 0.0668 & 0.1881 & 0.4502 \\
\hline \multicolumn{6}{|c|}{ Note: Values for HC (moderator) are the mean and plus/minus one standard deviation (SD) from mean } \\
\hline Mediator & $\mathrm{HC}$ & Effect & Boot SE & Lower & Upper \\
\hline $\mathrm{AC}$ & -1.3581 & 0.4261 & 0.0623 & 0.3146 & 0.5628 \\
\hline $\mathrm{AC}$ & -0.6497 & 0.3898 & 0.0589 & 0.2863 & 0.5203 \\
\hline $\mathrm{AC}$ & 0.0638 & 0.3533 & 0.0596 & 0.2507 & 0.4856 \\
\hline $\mathrm{AC}$ & 0.6185 & 0.3248 & 0.063 & 0.2162 & 0.4634 \\
\hline $\mathrm{AC}$ & 1.2783 & 0.291 & 0.0697 & 0.1702 & 0.4419 \\
\hline
\end{tabular}

Note: Values for HC (moderator) are 10th, 25th, 50th, 75th, and 90th percentiles

\begin{tabular}{lcccc}
\hline B) Index of moderated mediation & & & \\
\hline & & & \multicolumn{2}{c}{ BCCI } \\
\cline { 4 - 5 } Mediator & Index & SE (Boot) & Lower & Upper \\
\hline $\mathrm{AC}$ & -0.0512 & 0.0223 & -0.0994 & -0.01 \\
\hline
\end{tabular}

Notes: ISC: information systems capabilities; AC: absorptive capacity; OA: organizational agility; HC:

hierarchy culture. Control variables: Age and Size on OA. BCCI: Bias corrected confidence interval.

Bootstrapping based on $n=5000$ subsamples.

\section{Discussion}

Organizations must develop capabilities which connect them permanently to their environment and their stakeholders, and which enable them to transform captured information into rapid and precise responses. This paper has posited OA as a clear example of this type of mechanism. This study also contributes to enhancing the recent research on the firm's strategic efforts and endeavors to find mechanisms that lead to improving OA, proposing a model with ISC and AC as its antecedents. Firstly, we find support for the direct relationship between ISC and OA, a link that was not completely clear in the previous literature. Secondly, from a mediation point of view, we also provide evidence of the existence of an indirect effect of ISC on OA through AC. Results reveal that the influence of ISC on OA is more an indirect than a direct effect. This means that the firm's ISC will impact on OA enhancement concerning the extent to which it is capable of generating AC. Thirdly, we find support for the hypothesis suggesting the negative moderating role of $\mathrm{HC}$ on the AC-OA link.

This work presents some significant academic implications. First, while previous studies have suggested that ISC can enable organizational agility (Sambamurthy et al., 2003; Lu \& Ramamurthy, 2011), empirical evidence has been scarce, focused on partial aspects and lacking a framework to explain how ISC influence a firm's OA (Trinh-Phuong et al., 2012). This work tries to fill this gap, by developing and testing a comprehensive model, all of whose variables are defined in their most inclusive form of organizational capabilities.

Second, our results shed light on the existing gap concerning the potential impact of ISC on OA enhancement, and through which mechanisms they act. Furthermore, according to our results, we conclude that AC partially mediates the ISC-OA relationship

Thirdly results also indicate the counter effect of $\mathrm{HC}$ on the AC-OA link, acting as a moderator variable that decreases the direct link mentioned. Although the presence of cultural values associated with $\mathrm{HC}$ can hinder the positive effect of $\mathrm{AC}$ on $\mathrm{OA}$, the indirect relationship remains significant.

This negative moderating effect surprisingly contrasts with a non-hypothesized direct 
positive effect of $\mathrm{HC}$ on $\mathrm{OA}$. This result, which is theoretically quite controversial, might be explained by the fact that some of the characteristic values that shape this culture are in line with some of the attributes that customers and other stakeholders are demanding from firms within the current economic crisis scenario. In this sense, customers are nowadays challenging firms to satisfy their preferences while remaining efficient and controlling their costs. Such a situation may hence require patterns associated with an HC typology (i.e., the emphasis on standardizing processes, the stress on controlling, the bureaucratic approach, etc.) This finding opens up possibilities for further research related to the influence of external factors on the relationship between ISC and OA (Chen et al., 2014)

This study also has clear managerial implications. Our results reveal that, in order to enhance OA, organizations ought to improve their ISC while being able to develop their AC. Although the importance of ISC as an antecedent of OA has gradually gained recognition, how to develop and to put it into practice still remains uncertain. Our paper suggests that managers should foster and deploy the firm's knowledge absorption mechanisms to effectively maximize the impact of IS efforts and investments on achieving agility.

\section{Acknowledgement}

This research has been supported by the Junta de Andalucía (Regional Government of Andalusia) (Consejería de Economía, Innovación y Ciencia) Spain (Proyecto P10-SEJ-6081).

\section{References}

Alavi, M., Kayworth, T. R., \& Leidner, D. E. (2006). An empirical examination of the influence of organizational culture on knowledge management practices. Journal of Management Information Systems, 22(3), 191-224.

Ashrafi, N., Xu, P., Kuilboer, J. P., \& Koehler, W. (2006). Boosting enterprise agility via IT knowledge management capabilities. In Proceedings of the Annual Hawaii International Conference on System Sciences (Vol. 2, pp. 1-9).

Ashrafi, N., Xu, P., Sathasivam, M., Kuilboer, J. P., Koelher, W., Heimann, D., \& Waage, F. (2005). A framework for implementing business agility through knowledge management systems. In Proceedings - Seventh IEEE International Conference on ECommerce Technology Workshops, CEC 2005 Workshops (pp. 116-121).

Baron, R. M., \& Kenny, D. A. (1986). The moderator-mediator variable distinction in social psychological research: Conceptual, strategic, and statistical considerations. Journal of Personality \& Social Psychology, 51, 1173-1182.

Bharadwaj, A. S. (2000). A resource-based perspective on information technology capability and firm performance: An empirical investigation. MIS Quarterly, 24(1), 169-193.

Bradley, R. V., Pratt, R. M. E., Byrd, T. A., Outlay, C. N., \& Wynn, D. E. (2012). Enterprise architecture, IT effectiveness and the mediating role of IT alignment in US hospitals. Information Systems Journal, 22, 97-127.

Cameron, K., \& Quinn, R. (2011). Diagnosing and changing organizational culture: Based on the competing values framework (3rd ed.). San Francisco, CA: John Wiley \& Sons.

Cepeda-Carrión, G., Cegarra-Navarro, J. G., \& Jiménez-Jiménez, D. (2012). The effect of absorptive capacity on innovativeness: Context and information systems capability as catalysts, British Journal of Management, 23 (1), 110-129.

Charbonnier-Voirin, A. (2011). The development and partial testing of the psychometric properties of a measurement scale of organizational agility.M@n@gement, 14(2), 119-156.

Chen, Y., Wang, Y., Nevo, S., Jin, J., Wang, L., \& Chow, W. (2014). IT capability and organizational performance: the roles of business process agility and environmental 
factors. European Journal of Information Systems, 23, 326-342

Chin, W. W., Marcolin, B. L., \& Newsted, P. R. (2003). A partial least squares latent variable modeling approach for measuring interaction effects: Results from a Monte Carlo simulation study and an electronic-mail emotion/adoption study. Information Systems Research, 14(2), 189-217.

Cohen, W. M., \& Levinthal, D. A. (1990). Absorptive capacity: A new perspective on learning and innovation. Administrative Science Quarterly, 35, 128-152.

Cotec (2009). Tecnología e innovación en España. Informe COTEC 2009. Fundación Cotec: Madrid.

Crocitto, M., \& Youssef, M. (2003). The human side of organizational agility. Industrial Management \& Data Systems, 103, 388-397.

Doherty, N. F., \& Terry, M. (2009). The role of IS capabilities in delivering sustainable improvements to competitive positioning. Journal of Strategic Information Systems, $18(2), 100-116$.

Fornell, C. (1982). A second generation of multivariate analysis: an overview. In Fornell, C. (Ed.), A second generation of multivariate analysis (Vol. 1, pp. 1-21). New York, NY: Praeger.

Goldman, S. L., Nagel, R. N., \& Preiss K. (1995). Agile competitors and virtual organizations: strategies for enriching the customer. New York: Van Nostrand Reinhold.

Hair, J. F. J., Hult, G. T. M., Ringle, C., \& Sarstedt, M. (2014). A primer on partial least squares structural equation modeling (PLS-SEM). Thousand Oaks, CA: SAGE.

Hao, J. X., Yu, A. Y., \& Dong, X. (2011, July). Bridging role of absorptive capacity for knowledge management systems success. In Proceeding Papers. PACIS $2011-15$ th Pacific Asia Conference on Information (Article 73).

Hayes, A. F. (2009). Beyond Baron and Kenny: Statistical mediation analysis in the new millennium. Communication Monographs, 76, 408-420.

Hayes, A. F. (2013). Introduction to mediation, moderation and conditional process analysis. New York: The Guilford Press.

Hayes, A. F. (2015). An index and test of linear moderated mediation. Multivariate Behavioral Research, 50, 1-22.

Henseler, J. (2014). Common factor models, composite models, and formative measurement: Their nature, application, and testing. Lecture at University of Seville, November 20.

Henseler, J., Dijkstra, T. K., Sarstedt, M., Ringle, C. M., Diamantopoulos, A., Straub, D. W., Ketchen, D. J., Hair, J. F., Hult, G. T. M., \& Calantone, R. J., (2014). Common beliefs and reality about partial least squares: Comments on Rönkkö \& Evermann (2013). Organizational Research Methods, 17(2), 182-209.

Henseler, J., Ringle, C. M., \& Sarstedt, M. (2015). A new criterion for assessing discriminant validity in variance-based structural equation modeling. Journal of the Academy of Marketing Science, 43, 115-135.

Hu, L.-T., \& Bentler, P. M. (1999). Cutoff criteria for fit indexes in covariance structure analysis: Conventional criteria versus new alternatives. Structural Equation Modeling: A Multidisciplinary Journal, 6(1), 1-55.

Jansen, J. J., Van Den Bosch, F. A., \& Volberda, H. W. (2005). Managing potential and realized absorptive capacity: how do organizational antecedents matter? Academy of Management Journal, 48(6), 999-1015.

Liu, H., Ke, W., Wei, K. K., \& Hua, Z. (2013). The impact of IT capabilities on firm performance: The mediating roles of absorptive capacity and supply chain agility. Decision Support Systems, 54(3), 1452-1462.

Lu, Y., \& Ramamurthy, K. R. (2011). Understanding the link between information 
technology capability and organizational agility: An empirical examination. MIS Quarterly, 35(4), 931-954.

Malhotra, A., Gosain, S., \& Sawy, O. A. E. (2005). Absorptive capacity configurations in supply chains: Gearing for partner-enabled market knowledge creation. MIS Quarterly, 29(1), 145-187.

Nijssen, M., \& Paauwe, J. (2012). HRM in turbulent times: how to achieve organizational agility? The International Journal of Human Resource Management, 23, 3315-3335.

Overby, E., Bharadwaj, A., \& Sambamurthy, V. (2006). Enterprise agility and the enabling role of information technology, European Journal of Information Systems, 15 (2), 120-131.

Ravichandran, T., \& Lertwongsatien, C. (2005). Effect of information systems resources and capabilities on firm performance: A resource-based perspective. Journal of Management Information Systems, 21(4), 237-276.

Ringle, C. M., Wende, S., and Becker, J.-M. (2015). SmartPLS 3. Boenningstedt: SmartPLS $\mathrm{GmbH}$, http://www.smartpls.com.

Roldán, J. L., \& Sánchez-Franco, M. J. (2012). Variance-based structural equation modeling: Guidelines for using partial least squares in information systems research. In M. Mora, O. Gelman, A. Steenkamp, \& M. Raisinghani (Eds.), Research Methodologies, Innovations and Philosophies in Software Systems Engineering and Information Systems (pp. 193-221). Hershey PA: Information Science Reference.

Sambamurthy, V., Bharadwaj, A., \& Grover, V. (2003). Shaping agility through digital options: Reconceptualizing the role of information technology in contemporary firms. MIS Quarterly, 27(2), 237-263.

Sherehiy, B., Karwowski, W., \& Layer, J. K. (2007). A review of enterprise agility: Concepts, frameworks, and attributes. International Journal of Industrial Ergonomics, 37, 445460.

Tallon, P. P., \& Pinsonneault, A. (2011). Competing perspectives on the link between strategic information technology alignment and organizational agility: Insight from a mediation model. MIS Quarterly, 35(2), 463-486.

Teece, D. J., Pisano, G., \& Shuen, A. (1997). Dynamic capabilities and strategic management. Strategic Management Journal, 18(7), 509-533.

Trinh, T. P., Molla, A., Peszynski, K., Trinh-Phuong, T., Molla, A., \& Peszynski, K. (2012). Enterprise systems and organizational agility: A review of the literature and conceptual framework. Communication of the Association for Information Systems, 31, 167-193.

Wade, M., \& Hulland, J. (2004). Review: The resource-based view and information systems research: Review, extension, and suggestions for future research. MIS Quarterly, 28(1), 107-142.

Wright, R. T., Campbell, D. E., Thatcher, J. B., \& Roberts, N. (2012). Operationalizing multidimensional constructs in structural equation modeling: Recommendations for IS Research. Communications of the Association for Information Systems, 30(Article 23), 367-412.

Yang, C., \& Liu, H.-M. (2012). Boosting firm performance via enterprise agility and network structure. Management Decision, 50, 1022-1044.

Zahra, S. A., \& George, G. (2002). Absorptive capacity: A review, reconceptualization, and extension. Academy of Management Review, 27(2), 185-203.

Zammuto, R. F., Gifford, B., \& Goodman, E. A. (2000). Managerial ideologies, organization culture, and the outcomes of innovation: a competing values perspective. In N. M. Ashkanasy, C. P. M. Wilderon, \& M. F. Peterson (Eds.), Handbook of organizational culture and climate (pp. 261-278). Thousand Oaks, CA: SAGE. 